\title{
L'enseignement de la musique carnatique et son évolution
}

Jean-Paul Auboux

\section{(2) OpenEdition}

Journals

Édition électronique

URL : http://journals.openedition.org/ethnomusicologie/2303

ISSN : 2235-7688

Éditeur

ADEM - Ateliers d'ethnomusicologie

Édition imprimée

Date de publication : 1 janvier 1988

Pagination : 132-139

ISBN : 2-8257-0159-9

ISSN : 1662-372X

\section{Référence électronique}

Jean-Paul Auboux, "L'enseignement de la musique carnatique et son évolution », Cahiers

d'ethnomusicologie [En ligne], 1 | 1988, mis en ligne le 15 août 2011, consulté le 04 mai 2019. URL

http://journals.openedition.org/ethnomusicologie/2303 


\title{
L'ENSEIGNEMENT DE LA MUSIQUE CARNATIQUE ET SON ÉVOLUTION
}

\author{
Jean-Paul Auboux
}

Il peut sembler paradoxal d'écrire sur l'enseignement de la musique de l'Inde du Sud, car il est traditionnellement oral. Pourtant, cette ambiguïté est à l'image de la société indienne qui a évolué dans un équilibre, préservé jusqu'ici, entre la rigidité des écrits (les çāstra) et la mobilité de l'enseignement oral, entre le poids de la tradition et les mouvements éphémères des styles individuels, des courants d'écoles et parfois même des modes.

\section{La musique dans la société}

L'enseignement musical en Inde reflète, lui aussi, la société qui l'a fait naître. A travers son évolution, on peut suivre celle des grandes tendances de la société indienne, de ses perméabilités et résistances au contact d'autres civilisations. Il faut noter à ce titre que, s'il y eut de nombreux échanges et des influences de cultures très diverses dans le passé (Grèce, Chine, etc.), c'est surtout la société dite occidentale et moderne qui semble avoir secoué les piliers de la tradition.

Puisque l'Inde du Sud est restée géographiquement et culturellement protégée des multiples invasions qui ont touché le nord du subcontinent, même sous domination anglaise, ce sont bien les nouvelles techniques et les modes de vie liés aux systèmes modernes de communication qui transformèrent la société plus que jamais.

Historiquement, le premier ouvrage où apparaît le mot karnātaka est le très ancien brhaddeçī de Sarngadeva. Mais c'est dans le sañīta-sudhākara de Haripala, écrit au début du XIV ${ }^{e}$ siècle, que se trouvent les premières références écrites aux mots "hindustani» pour le système musical de l'Inde du Nord et «carnatique» pour celui de l'Inde du Sud. Il est généralement admis que c'est avec les invasions islamiques et plus particulièrement sous l'influence de la dynastie mongole timuride dite moghole, qui régna à partir du $\mathrm{XVI}^{\mathrm{e}}$ siècle, que la scission entre les deux systèmes musicaux devient significative.

En tamoul, le terme de karnātaka, outre qu'il indique l'Etat du même nom, signifie «pur» ou «traditionnel». On appelle karnătaka-manusya un homme dont le mode de vie est conforme aux coutumes anciennes, ce qui en dit long sur ce que pensent les Indiens du Sud de leur système musical. 


\section{La méthode traditionnelle d'enseignement de la musique carnatique}

C'est à l'époque de l'implantation musulmane que Purandaradasa codifia l'enseignement de la musique carnatique sous forme d'exercices musicaux et de compositions diverses, destinés à la mémorisation musicale progressive et pratique des diverses théories existantes. L'apprentissage musical devint dès lors différent dans le nord et le sud : le rāga de base sur lequel l'étudiant fera ses premières gammes sera mayamalavagaula: tonique, seconde mineure, tierce, quarte, quinte naturelles, sixte mineure, septième naturelle ${ }^{1}$.

Depuis des siècles, les exercices très précis de Purandaradasa servent sans changement à assouplir la voix, le cerveau et les doigts des instrumentistes, tandis que des compositions de difficulté croissante permettent l'assimilation progressive de formes musicales plus complexes et de rāga et tâla nouveaux:

- Les gïtam sont les premières compositions que l'élève apprend. Les mélismes en sont absents, du moins dans l'écriture, et la mélodie en est simple ; par contre, l'étudiant y aborde déjà sous une forme musicale l'application de la théorie complexe de la classification des rāga et des tāla.

- Puis ce sont les jätisvaram et les svarajāti: figures au dessin mélodique plus complexe, qui sont aussi utilisées dans les danses classiques bharatanätyam et mohiniyāttam.

- Viennent ensuite les varna: compositions très denses où doivent figurer tous les aspects et toutes les couleurs d'un rāga. Les varna sont destinés à l'enseignement et permettent à l'étudiant d'avoir une idée précise du rāga à travers ses principaux prayoga (phrases spécifiques, particulières à ce rāga et virtuellement obligées dans son exposition), tout en apprenant une composition. Les varna sont extrêmement difficiles, et seuls les grands musiciens se sont permis d'en composer.

Les varna se retrouvent dans les récitals de danse classique, mais sous une forme très développée, puisque c'est généralement le morceau central et le plus élaboré du récital. La coutume veut aussi que les concerts de musique commencent par un varna pour "mettre en forme" le musicien et son public.

- L'étude se termine par les krti (ou kirtana). C'est au siècle dernier que Tayagaraja donna à ce type de composition sa forme actuelle, considérée depuis lors comme parfaite. D'un point de vue pédagogique, les $k r t i$ permettent une étude approfondie des rāga et des élaborations complexes basées sur des découpes rythmiques ou mélodiques. Celles-ci, à travers les sarigati et les nirraval, ouvrent la voie aux développements savants des pallavi. Puis ce sont les padam et les javali qui, eux, sont difficiles en raison de leur simplicité même ${ }^{2}$ : c'est après les calculs savants, le retour à l'émotion pure.

\section{L'enseignement oral et écrit}

En associant presque toujours tradition et oralité, on oublie souvent que l'oralité fait plus précisément référence à la manière dont s'effectue la transmission du savoir; or, bien que l'Inde soit souvent citée comme l'exemple d'une société de tradition orale, elle n'en connaît pas moins l'écriture 
depuis la plus haute antiquité, et il faut considérer ses écrits comme étant complémentaires de la transmission orale. A cet égard, il serait d'ailleurs souhaitable de définir les frontières entre ce qui relève de l'oral et ce qui appartient à l'écrit.

Avant les çāstra et l'invention de l'écriture, la transmission orale était aussi précise que l'écrit, surtout dans des domaines auxquels l'écriture n'avait pas accès : en témoignent de nos jours encore les récitations rituelles des veda, enseignées oralement, toujours chantées selon leur scansion originelle entêtante. Le parfum tellurique et immémorial qui se dégage de ces récitations, ainsi que la gestuelle, la mise des officiants et le rituel tout entier n'ont pas changé depuis des millénaires. D'autre part, il ne serait pas inutile d'adopter, pour la civilisation indienne, une définition plus large de l'écrit qui engloberait alors tout ce qui est fixé matériellement ${ }^{3}$. De même, pour citer le grand maître T.R. Mahalingam qui disait que les quatre-vingt-dix-neuf pour cent de l'enseignement passent à travers l'écoute, on peut concevoir les enregistrements de concerts sous forme de cassettes ou même mémorisés, comme une forme d'écriture. Ce serait encore plus sensible dans le cas de disques: il n'en existe malheureusement aucun, à moins qu'il ne s'agisse d'enregistrements de concerts publics, où la musique censément improvisée n'est pas pensée et décidée avant l'enregistrement. Cela s'explique par le fait qu'un Indien considère un enregistrement comme l'équivalent d'un écrit: il doit être formellement parfait, exigence qui passe avant toute considération de fluidité d'inspiration, d'expression et de jeu.

Cette fluidité est pourtant à la base même de la musique carnatique qui, selon les çāstra, doit couler "comme coule l'huile que l'on verse sur la lampe ». Le mieux serait-il l'ennemi du bien en création musicale? Y a-t-il
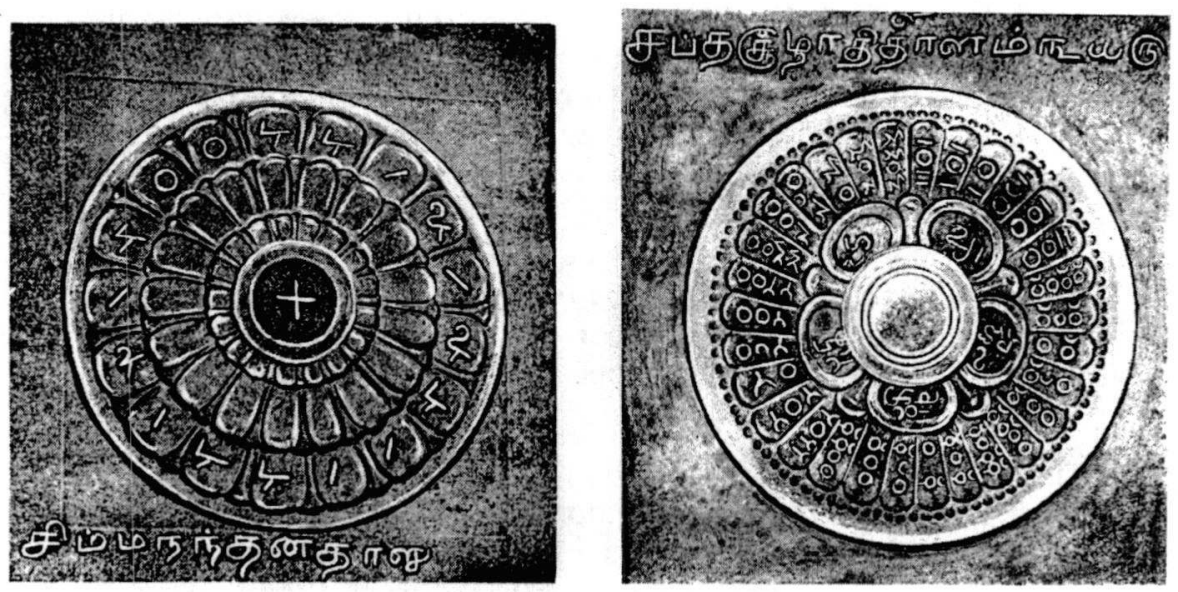

Sculptures de tāla au temple de Śrī Mīnakśi Amman, Madurai, Inde. 
même un critère de perfection et en quoi consiste-t-il ? Même une oreille non avertie remarque immédiatement la différence entre une musique inspirée et improvisée spontanément et une musique décidée d'avance.

\section{La «saveur subtile » de la musique carnatique}

La musique est habituellement classée en deux grands systèmes : l'harmonique et le mélodique. La musique carnatique fait partie du système mélodique et, comme c'est le cas de la majorité des musiques de ce type, l'enrichissement musical se produit grâce à de très fines modulations qui échappent à l'analyse, même si un certain nombre d'entre elles sont nommées et codifiées. Tout l'art du musicien consiste précisément à introduire dans sa musique ce parfum indéfinissable que les Indiens du sud apprécient tant et qu'ils nomment bhāva. Cette "saveur subtile» est en grande partie due à la finesse et à la sensibilité avec lesquelles le musicien développe ces modulations. Les "notes cachées» (nokku-svara) que l'oreille perçoit sont-elles glissées, étirées? Aucune notation ne peut en rendre compte fidèlement, tant leurs paramètres sont complexes et leur exécution rapide. C'est pourquoi la notation musicale ne constitue qu'un aide-mémoire, précieux certes, mais en tout cas insuffisant pour connaître une nouvelle composition si elle n'a pas été entendue auparavant.

Il y a là une différence fondamentale entre les systèmes occidental et carnatique : en Inde du Sud, la théorie est religieusement respectée et ne laisse aucune place à la contradiction. Quant aux morceaux écrits (qui n'ont rien à voir avec la sophistication d'une partition de musique occidentale), ils présentent une telle distance entre le signe et l'exécution qu'ils laissent supposer de très grandes différences d'interprétation au cours des siècles.

\section{Le maître}

De tout temps, la civilisation indienne a insisté sur l'importance du contact humain dans la transmission du savoir, sans doute parce qu'elle situe l'homme au centre de toute connaissance. Cela s'applique à la musique comme à tous les autres domaines : le maître est appelé guru, le système luimême étant désigné par le terme de gurukula. Ici également, ce dernier terme comporte une acception spirituelle, car la musique est sans doute un miroir subtil, permettant de comprendre en profondeur les tendances et les accomplissements tangibles d'une culture où la musique, la société et la religion entretiennent depuis des millénaires des liens étroits et concourent dans la quête d'une vision globale, embrassant l'unité ultime, la source et la finalité de tous les aspects de la vie.

La musique sollicite aussi bien les sens que l'esprit, touchant ainsi à tous les éléments de la perception esthétique. Parce qu'il est vivant, le contact humain encourage ici une assimilation virtuellement mimétique qui, au-delà du style, permet d'absorber une personnalité, voire même une âme. C'est un élément essentiel de la musique carnatique: vu l'importance de l'improvisation, on écoute lors d'un concert autant la musique que le musicien, même lorsque celui-ci interprète une composition. 
Le rapport entre l'apprentissage auprès d'un maître et l'écoute de la musique de concert fixe les deux pôles de l'enseignement musical. L'aspirant musicien devra écouter énormément de musique. Mais pour le faire intelligemment et surtout pour retenir ce qu'il a entendu, il lui faut généralement l'aide d'un maître. Pour reprendre l'expression de T.R. Mahalingam, «le un pour cent d'instruction directe auprès d'un maître est aussi important que les quatre-vingt-dix-neuf pour cent restants: l'oreille du maître qui écoute et corrige n'est en aucun cas comparable à celle de l'élève». T.R. Mahalingam veut surtout indiquer par là que les techniques actuelles d'enregistrement permettent de travailler en écoutant et en réécoutant les grands maîtres. C'est la seule technique d'absorption musicale qui, de tout temps, a formé les musiciens, parallèlement à un enseignement qui permet de distinguer, de classer, de trier et de comprendre ce qui a été entendu, puis de le reproduire, si on y parvient. Ce qui aurait demandé des jours, des semaines ou des mois d'attente au disciple attentif est maintenant possible immédiatement, grâce aux enregistrements ${ }^{4}$.

L'accès, désormais sans contrainte, aux enregistrements des grands maîtres, par la cassette ou le disque, a sans doute modifié l'enseignement luimême, en libérant le maître d'une partie de cette tâche répétitive, nécessaire tant que l'élève ne maîtrise pas telle ou telle subtilité.

Ainsi, pour la tierce mineure du räga-todi (à ne pas confondre avec le rága-todi du système hindustani), on peut distinguer au moins cinq types d'exécution qui vont du mouvement oscillant entre la seconde majeure et la quarte avec un écrasement et une insistance dans la durée et l'intensité sur la seconde, jusqu'à une très légère oscillation autour d'une tierce mineure surbaissée frôlant la seconde majeure. Semblables aux "tours de main" caractéristiques des métiers manuels, ces formes subtiles, «tours de main mélodiques ", sont difficiles à saisir : elles exigent le contact avec un maître patient et prêt à enseigner son art dans le détail.

Il n'est pas aisé, au demeurant, d'établir un lien entre une théorie nette et assimilable sans trop de peine, et une pratique qui fait plonger l'élève dans une réalité musicale qui semble venir d'un autre monde. La question se pose de savoir si la musique relève du domaine du volontaire ou de l'involontaire ; mais l'aspiration commence là où s'arrête la volonté. C'est pourquoi la tradition carnatique fixe très loin les bases et les limites du volontaire, en permettant à l'inspiration de prendre son essor à un niveau insaisissable par tout autre moyen.

\section{L'enseignement de nos jours}

Le cursus est donc précis et, semble-t-il, immuable ; sa fixation le rend assimilable aux çāstra et virtuellement sacré pour l'Indien accoutumé à vénérer parfois des écrits pour leur simple ancienneté. Dans le contexte des mutations sociales de la seconde moitié de ce siècle, cette fixité a certainement pu justifier et encourager les récentes tentatives pour créer un enseignement de type occidental, quoique inadapté à la nature même de la musique indienne. 

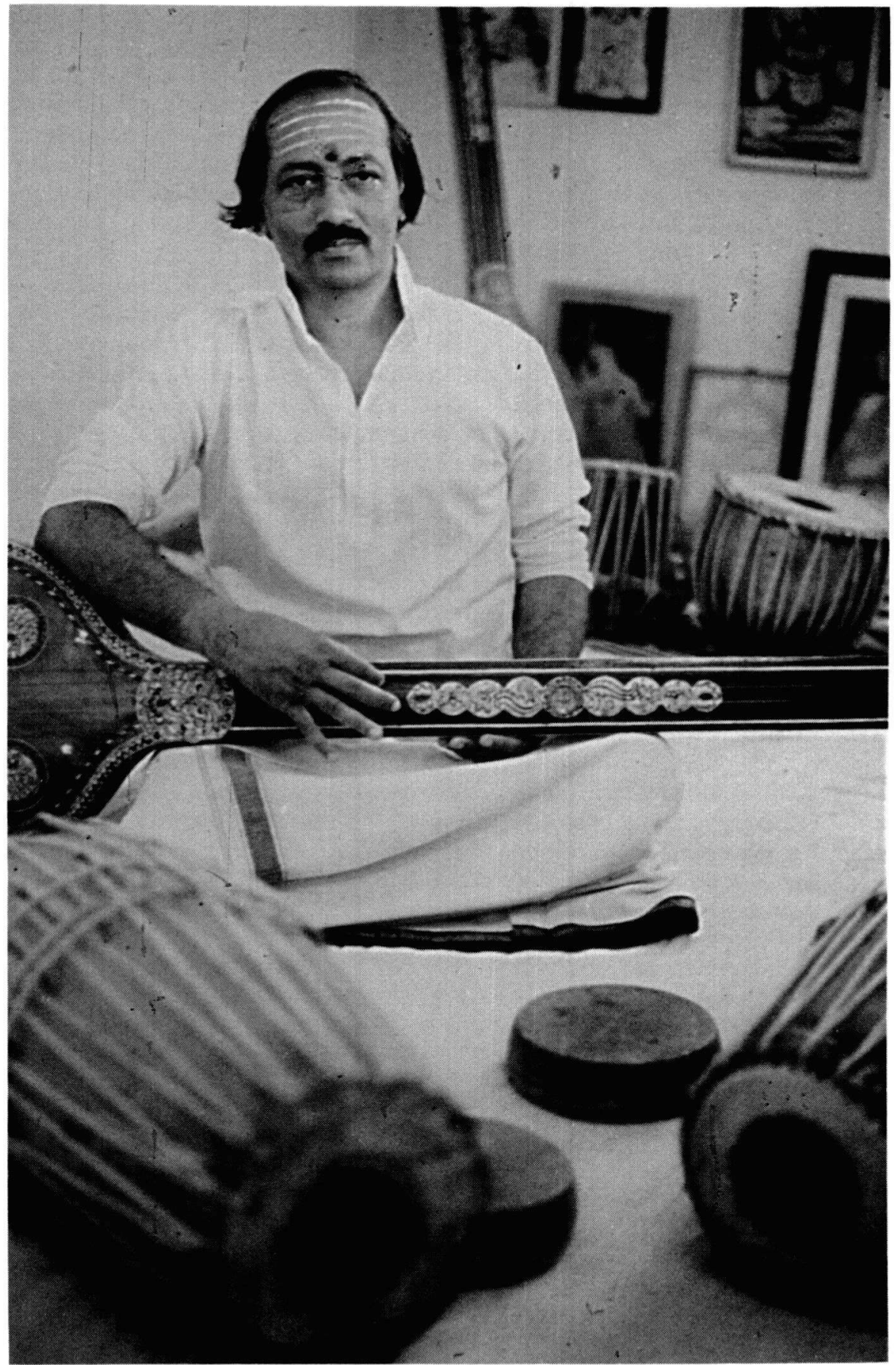

Le chanteur et percussionniste T.V. Gopalkrishnan (photo : Raghavendra Rao). 
C'est donc au cours des trois dernières décennies que des changements majeurs sont survenus: le système dit gurukula lui-même en est atteint. Plus qu'une forme, c'est un état d'esprit qui s'est transformé. Ce qui était un échange humain devient un commerce, s'il ne se fige pas dans des institutions. En effet, tandis qu'on assiste à une démocratisation du système de rémunération des musiciens, une tendance semblable se fait sentir dans l'enseignement musical: des tentatives sont faites pour créer des écoles de musique sur le modèle de nos conservatoires. Remarquons à ce sujet que les sabhā modernes, c'est-à-dire les associations qui organisent des concerts payants, sont considérés comme l'expression d'une démocratisation de la culture par opposition à l'ancienne coutume des maharajas d'entretenir des musiciens de cour et d'offrir en contrepartie des concerts publics gratuits. En donnant la possibilité à ses musiciens d'être rétribués par leur auditoire, le peuple indien a privé les plus pauvres de l'accès aux concerts, si ce n'est par la radio. Ce qui est décrit paradoxalement comme une démocratisation de la culture.

La prétendue démocratisation de l'enseignement musical produit, quant à elle, des effets pareillement pervers : le désir de créer des écoles calquées sur les conservatoires occidentaux afin d'ouvrir l'enseignement au plus grand nombre risque en fait - et c'est bien ceci qui semble se passer actuellement d'en faire baisser dramatiquement le niveau et, ce qui est peut-être plus grave encore, d'empêcher certains élèves d'y accéder. Les écoles ont des exigences financières, alors que l'ancien système dit gurukula, s'il impliquait des notions de service et de don, n'entraînait jamais de transaction financière ; cet état d'esprit risque malheureusement de s'infiltrer et de perdurer tout au long d'une "carrière" que l'on imaginerait purement musicale. Elle serait alors bien loin des buts avoués de la vraie musique qui, de tout temps et en tous lieux, a été un mode d'être, un mode de vie.

Restons cependant convaincus que les nobles idées exprimées par les grands compositeurs de musique carnatique, qui continuent d'être chantés de nos jours, garderont vivante «une certaine idée» du Vrai et du Beau, qui était leur inspiration et leur but.

\section{Notes}

\footnotetext{
${ }^{1}$ Le choix de ce räga est typique d'une certaine tournure d'esprit organisatrice et logique, caractéristique de l'Inde du Sud. On y trouve toutes les symétries, naturelles, renversées et de tétracordes, ainsi que les trois intervalles, demi-ton, ton et tierce mineure, fréquents dans la musique carnatique.

${ }^{2}$ La différence entre les pallavi et les padam illustre bien les deux grandes constituantes de la musique carnatique que l'on retrouve dans l'adage immémorial çruti mätāa, laya pitā (la justesse de ton est la mère, et l'exactitude rythmique est le père). D'une part, l'aspect mental et savant de la rythmique qui est une mathématique vécue et poussée à ses limites. Il semble
} 
d'ailleurs qu'aucune autre musique ne soit allée aussi loin dans la complexité des développements mathématiques de sa rythmique: les pallavi ou les développements de nirraval à l'intérieur des $k r t i$ en témoignent. D'autre part, l'aspect émotionnel qui traverse la fluidité et le mouvement d'une mélodie apparemment simple et dont la magie ne "tient qu'à un fil». C'est là le propre des padam et des javali. Le flûtiste T.R. Mahalingam est le seul musicien qui ait su faire chanter l'un aussi bien que l'autre aspect de la musique carnatique.

${ }^{3}$ L'écriture peut même prendre la forme d'un bas-relief : une iconographie du savoir et de la pratique des arts musicaux et de la danse abonde dans les temples de l'Inde du sud. Voir les illustrations ci-jointes, qui montrent le cycle des tâla ainsi que l'organisation des différentes parties du très complexe simhanandana-tāla, gravés sur la pierre. On est en droit de penser que les sculptures si décoratives de danseuses ou de musiciens qui ornent les façades des temples étaient au moins autant destinées à la transmission d'un savoir qu'à des fins purement esthétiques.

${ }^{4}$ T.R. Mahalingam insistait sur cet aspect de l'enseignement "d'oreille», car c'est ainsi qu'il s'était formé lui-même. C'était un génie musical et sans doute le seul instrumentiste à avoir appris tout seul en écoutant et en travaillant intensément la musique carnatique. 